\title{
Los museos de etnología en Europa: entre la redefinición y la transformación ${ }^{1}$
}

\author{
Agustí Andreu Tomàs ${ }^{2}$ \\ Universidad Rovira i Virgili, Tarragona, España \\ E-mail: agusti.andreu@urv.cat
}




\section{Resumen}

En esta comunicación se analiza el proceso de redefinición de los museos de etnología en Europa, caracterizado por una recontextualización del objeto etnológico y por una profunda renovación museográfica:

La recontextualización del objeto etnológico afecta tanto a los museos de temática "exótica" como a los que contienen el patrimonio de la cultura local. Aquellos museos que han constituido su fondo a partir del típico modelo colonialista de la sociedad occidental, se han visto obligados a redefinir su papel. La mayoría de estos museos se han convertido en un espacio de dialogo y reconocimiento entre culturas, se orientan hacia una reflexión sobre el multiculturalismo y las relaciones interculturales. Otros, en cambio, han optado por reconvertir el objeto etnológico en obra de arte y han apostado por una desvalorización de los componentes sociales y culturales de los objetos en beneficio de sus cualidades estéticas o de sus aspectos técnicos.

Palabras clave: Patrimonio etnológico. Museología. Museos de civilización. Museos de sociedad.

\section{Abstract}

This paper analyses the process of redefining ethnological museums in Europe, which is characterized by a re-contextualization of ethnological objects and a profound renewal of the museums.

The re-contextualization of ethnological objects has occurred in both museums containing "exotic" exhibits and those that contain local cultural artefacts. Those museums that were created on the basis of the typical colonialist model of Western society have been obliged to redefine their role. Most of these museums have become a space for dialogue and recognition between cultures and are oriented towards a reflection on multiculturalism and intercultural relations. Others, however, have chosen to turn their ethnological objects into works of art and have opted to devalue the social and cultural components of these objects in favour of their aesthetic qualities or its technical aspects.

Keywords: Ethnological heritage. Museology. Museums of society and civilization. 
$\mathrm{E}$ n relación al origen de la palabra museo, el museólogo belga François Mairesse, constata que la história de esta institución comienza con una paradoja relacionada con la idea de colección. Según este autor todas las tareas inherentes a la concepción moderna de museo (la adquisición, la conservación, la investigación, la comunicación,...) están condicionadas por la colección. La paradoja la encontramos en el hecho de que el mouseion griego, al que debemos el origen etimológico del la palabra museo, no tiene, practicamente, ninguna relación con la idea de colección. La acepción antigua de museo está relacionada con la idea pitagoriana de culto a las musas, con un claro papel de discusión filosófica y como lugar donde se reúnen los sabios. De hecho el museion de Alejandría es descrito por Strabon (citado por Mairesse, 2002, p. 14-15): como un gran edificio donde se localiza la sala comunitaria en la que los sabios, miembros del mouseion, comen. Strabon no habla de colecciones y por otra parte los arqueólogos e investigadores contemporáneos tampoco han podido probar la hipótesis de la colección. En cambio, todos están de acuerdo en que el aspecto principal del museion es el estudio filosófico.

Para reforzar esta idea Mairesse cita a G. Roux, que en un artículo del año 1954, estudió otro prototipo de mouseion, el M. del Helicón. Roux comenta que el ambiente del mouseion permitía el clima necesario para el nacimiento de la inspiración y el desarrollo del pensamiento. Tanto en Alejandría como en Helicon está totalmente constatada la existencia de bibliotecas y de objetos pero estos no constituyen de ninguna manera el aspecto central de la institución. La relación entre colección y museo se empieza a articular en el Renacimiento.

A partir del Renacimiento el coleccionismo se convertirá en el punto de partida de la construcción social del patrimonio y de la apa- 
rición del museo moderno. Junto con la idea de colección, el museo moderno se caracteriza por ser una institución pública. El siglo XVIII es el siglo de la aparición del museo moderno como consecuencia de la estatalización de las grandes colecciones privadas. El museo pasa de un estatus privado a un estatus público. El museo público es, pués, una iniciativa del Estado. Desde la segunda mitad del siglo XVIII algunas de las colecciones privadas iniciadas en el Renacimiento pasan a formar parte del patrimonio nacional y dan lugar a los grandes museos nacionales. Uno de los primeros museos abiertos al público fue el Ashmolean Museo de Oxford (1683) que proviene de la colección de John Tradescant, formada básicamente por instrumentos científicos y especímenes naturales. También cabe destacar que en este mismo país, en 1753, un Decreto del Parlamento creó el British Museum (inaugurado en 1759). En este salto cualitativo que se produjo en siglo XVIII jugó un papel muy importante la Revolución Francesa. La Asamblea Legislativa y la Convención promulgaron, entre 1792 y 1794, varios decretos por los que se crearon cuatro museos nacionales.

\section{El origen de los museos de etnología: el patrimonio de los otros}

En relación a la creación de los museos etnográficos (siglo XIX) hemos de comentar que su orígen esta relacionado con varios factores: con el desarrollo de los movimientos folkloristas, con el proceso de naturalización del mundo que se da en esta época, con la multiplicación de los viajes de exploración y, también, con las grandes conquistas coloniales. Por otro lado, tampoco nos hemos de olvidar de la importancia que tuvieron en la creación de los museos etnográficos los llamados "gabinetes de curiosidades. Varios autores (entre ellos Van Gennep y Rivière) nos sitúan la aparición del objeto etnográfico en el período en que surgen este tipo de gabinetes. La, digamoslo así, obsesión por coleccionar se ampliará a las clases medias, lo que implicará una diversificación del tipo de coleccionista, unos cambios en el estatus social y económico de quien colecciona y, también, unos cambios en el contenido de lo que se colecciona. Es el periodo en el que, junto 
con las naturalia y realia, se establecen otras dinámicas, las rariora, las curiosa y las mirabilia.

Estos gabinetes, que coleccionan los objetos de la naturaleza y los creados por el hombre en función de su rareza, su carácter insólito o fabuloso se convertirán en el punto de entrada del que más adelante será considerado como objeto etnográfico y algunos de estos gabinetes de curiosidades serán la base de la futura creación de museos de etnografía. En este sentido el caso posiblemente más paradigmático sea el de la actual Musée de Ethnographie de Neuchâtel en el origen del cual encontramos el "Cabinet d'Histoire Naturelle et de Curiosités Ethnographiques" del General Charles-Daniel de Meuron.

Los gabinetes de curiosidades, al ordenar las colecciones por criterios de curiosidad o excepcionalidad, poco tienen que ver con la manera de trabajar de los que serán los futuros museos de etnografía. El paso del gabinete de curiosidades al museo etnográfico implicará pasar de un coleccionismo preocupado por mostrar lo bizarro e inhumano de las costumbres de determinadas sociedades a un sistema de clasificación que, al igual que la antropología, intentará poner de relieve la existencia de unos estadios evolutivos culturales y demostrar científicamente, a partir de los objetos, la superioridad de unas culturas sobre las otras en base a la dicotomia primitivos/civilizados.

Hemos de comentar que la "revolución" que supuso la aparición de la consideración del objeto de colección como fuente de conocimiento ya se inició dentro mismo de algunos de estos gabinetes de curiosidades. El tratado de museología más antiguo (Mairesse, 2002) es el del médico Samuel Quiccheberg (1565). En este tratado el museo es considerado como un lugar donde hay cosas espectaculares o extraordinarias que deben estar accesibles para su estudio y admiración. Quiccheberg clasifica los objetos del museo en cinco clases subdivididas en 10 u 11 subclases. El objeto es entendido como fondo: como fondo de archivo y como fondo de colección. La idea de fondo nos remite a un objeto que puede ser estudiado o expuesto y, mientras tanto, es conservado y este proceso es el principio básico del museo moderno. Otro ejemplo de esto que estamos comentando lo constituye el gabinete de curiosidades de Ulisse Aldrovandi. Este gabinete nos muestra una 
faceta a menudo olvidada de este tipo de coleccionismo, la acepción de la noción de curiosidad como microcosmos o resumen del mundo donde encuentran su lugar los objetos de los tres reinos (mundo animal, vegetal y mineral) junto a las producciones del hombre. Aldrovandi (1522-1605) no sólo acumula objetos sino que los clasifica, los describe y los utiliza por sus tratados, es decir el objeto forma parte de un dispositivo de construcción de un saber científico

En esta construcción naturalística del objeto etnográfico cabe destacar el papel que desarrolló Edme-François Jomard, conservador del "Dêpot géographique de la Bibliothèque Royale". Jomard centró sus esfuerzos en la creación de una colección especial destinada a recibir los productos de los viajes lejanos y, en sus planteamientos, la etnografía es considerada como una ciencia auxiliar de la geografía, que nos debe permitir conocer las costumbres y los usos de las naciones y los pueblos exóticos. En un documento elaborado en 1831, nos comenta lo siguiente:

[...] a faire connaître, d'une manière exacte et positive, le degré de civilisation des peuples peu avances dans l'echelle sociale, en donnant le moyen d'aprécier leurs ouvrages, et jetant une vive lumière sur l'etat de leurs arts et de leur économie domestique, autant que sur la nature de leurs idees morales et religioses (Jomard citado por Hamy, 1988, p. 129).

En este mismo documento del año 1831, Jomard hace referencia a dos de los aspectos que, incluso hasta nuestros días, caracterizarán el patrimonio etnológico 1 ) el objeto testimonio y 2) la idea de salvamento etnográfico. La colección etnográfica implica que los objetos deben ser considerados en relación a su utilidad práctica y social, destacando tres dimensiones: su uso económico, su dimensión técnica y sus aspectos simbólicos. Jomard justifica, también, esta colección de etnografía en función de los cambios que en estas sociedades está provocando el contacto con el hombre blanco. Una justificación idéntica la encontraremos en el momento que el patrimonio etnológico se amplía a la cultura popular y, por otra parte, es bien conocido que nuestra disciplina siempre adolece de este carácter de urgencia: analizamos los rasgos culturales que están a punto de desaparecer. 
Otro de los aspectos interesantes de la obra de Jomard está relacionada con la introducción de un nuevo orden en el museo, en este sentido cabe destacar la correspondencia mantenida con Phillip Franz von Siebold médico de origen alemán que, como médico militar , fue destinado a Japón en los años 20 del siglo XIX.Aunque el interés específico de von Siebold fue la flora y fauna japonesa también reunió, en sus 7 años de estancia en Japón, una notable colección de objetos etnográficos (museo de Leiden-1831). En los años 40 del siglo XIX la correspondencia mantenida entre ambos personajes se centra, básicamente, en el sistema clasificatorio que debe regir la ordenación de las colecciones. Von Siebold era partidario de un sistema geográfico, por procedencia, en la clasificación de los objetos mientras que Jomard era partidario de una clasificación por materias. En su plan de clasificación por materias, Jomard, sólo contempla aquellos objetos trabajados por la mano del homme, el objeto etnográfico excluye las producciones naturales (la naturaleza bruta) y las de la Europa civilizada. Su sistema es tipológico, cada clase se divide en órdenes, cada orden se divide en géneros y cada género en especie (la materia, la forma, las dimensiones, el lugar de donde provienen los objetos constituye la especie). El objeto etnográfico encuentra, pues, su sentido en una naturalización de la vida social.

Los esfuerzos de Jomard para crear una colección de etnografía culminarán con la inauguración del Museo de Etnografía de Trocadero en 1878, con Ernest-Théodore Hamy como conservador del primer museo de etnografía en Francia. El sistema de clasificación de Hamy combinaba la clasificación de Jomard siguiendo la naturaleza y el uso de los objetos con la aproximación en términos de área geográfica de von Siebold.

Unos años más tarde, hacia finales del siglo XIX, en este caso en Inglaterra y Estados Unidos, se produce la consolidación del modelo tipológico en los museos de etnografía. Uno de los mejores ejemplos lo encontramos en el Museo Pitt-Rivers. Este militar, antropólogo y arqueólogo se dedicó a coleccionar objetos de la vida cotidiana de diferentes culturas organizando su presentación en base a unos criterios tipológicos en donde objetos estaban clasificados en función de sus 
cualidades formales y funcionales. La cultura material de diferentes periodos y sociedades se presentaban juntos en un intento de mostrar la evolución de lo simple a lo complejo y de representar una reconstrucción de la historia del mundo a partir de la dicotomía primitivo/ civilizado, convirtiendo el museo en un claro ejemplo de etnología comparada.

A partir de Pitt-Rivers y, sobre todo, con Boas el museo se convertirá en uno de los principales ámbitos de profesionalización de la antropología y la cultura material fue una herramienta de apoyo de las teorías antropológicas. Boas y su particularismo histórico introdujeron en el museo una nueva manera de hacer, una nueva manera de concebir este objeto de conocimiento. A partir de ahora el objeto no debía ilustrar un período de desarrollo, a partir de ahora el objeto debía representar una cultura. La cultura era, pues, el sistema que daba sentido al objeto etnográfico y, en consecuencia, la colección tenía que presentar la vida de una tribu.

La antropología relativista entendía que se debían presentar culturas, totalidades culturales, es decir, tenía como misión el restituir un estilo de vida. Para el particularismo histórico los objetos creados por una cultura son portadores de significación y no indicadores de un estadio cultural. Este interés por la contextualización hizo que la museografía relativista buscase nuevas fórmulas de representación de los objetos a fin de conseguir un mayor realismo en su contextualización. Una de estas fórmulas fueron los "living groups", grupos de maniquis que representaban escenas de la vida de las diferentes unidades culturales sobre las que trabajaba el museo.

Según Boas el museo debía cumplir con tres funciones básicas: entretenimiento, investigación y educación. Desde este punto de vista los objetos se agrupaban en dos series diferentes: objetos de exposición (entretenimiento/educación) y objetos de colección (investigación). Los objetos de colección eran una herramienta de investigación, una herramienta de instrucción universitaria. El museo era un laboratorio de antropología. El entusiasmo de Boas por los museos, pronto dejó paso a un escepticismo fundamentado en el hecho de considerar que la cultura material ofrecía toda una serie de limitaciones a la hora de comunicar los conceptos generados por la investigación antropológica. 
Desde Boas la antropología optaría por la escritura como medio de traducción de la experiencia científica. El particularismo histórico, junto con el funcionalismo, fusionarán el etnógrafo (descriptor de costumbres) y el antropólogo (constructor de teorías) y provocarán la definitiva constitución del método etnográfico a través del trabajo de campo y, por tanto, la relación directa del antropólogo con el informante. Este hecho incidirá en el progresivo alejamiento de la disciplina del mundo de los museos y su ubicación en la Universidad.

Finalizada esta época de oro de los museos, el patrimonio etnológico tendrá un nuevo impulso a partir de la creación de una serie de museos, aparecidos a finales del siglo XIX, que se ocuparán de la cultura popular.

\section{De los otros al nosotros: museos de etnología y cultura popular y tradicional}

De la misma manera que la antropología dejará de dedicarse exclusivamente a las llamadas sociedades primitivas y los antropólogos iniciarán trabajos de campo en su propia sociedad, el museo etnográfico posara su mirada sobre su entorno más inmediato y será necesaria una redefinición de la concepción del objeto etnográfico. A partir de ahora el objeto etnográfico ya no será sólo aquel objeto de una sociedad lejana y de la mano, en un primer momento, del folclore se ampliará a la cultura popular. La extensión del patrimonio etnológico a la cultura popular tuvo un gran impulso con la creación de los museos al aire libre escandinavos, los heimatmuseos alemanes y, posteriormente, con los museos de artes y tradiciones populares.

A finales del siglo XIX se produce un hecho crucial en la historia de los museos. A partir de esta fecha el museo inicia el camino que le llevó a la superación de uno de los problemas que aún tenía pendientes: la vieja polémica sobre si estas instituciones deben dedicarse exclusivamente a los grandes acontecimientos de la humanidad y, consecuentemente, ignorar por sistema los hechos micro sociales. El triunfo de la perspectiva universalista (Clair, 1972) implicará la incorporación en las salas de los museos, junto a las obras de arte y los testimonios de una cultura científica, los objetos de la vida cotidiana 
y de las clases más desfavorecidas. Los museos de etnología dejarán de ser un museo de lo exótico, del patrimonio de las otras culturas, las culturas llamadas primitivas, para incorporar en su discurso la temática de la cultura popular. A partir de este momento el museo etnogràfico activará el patrimonio en base a los siguientes criterios:

1. Activación patrimonial a partir de una tendencia, de reminiscencias claramente románticas, de recuperación del que se está perdiendo. Entre otras consideraciones, se pretende conservar el conocimiento sobre lo que desaparece (la cultura popular y tradicional) por influencia de la sociedad industrial y contribuir así al mantenimiento de la diversidad cultural.

2. Activación patrimonial persigue que poner de relieve la diversidad cultural de un determinado territorio y favorecer la emergencia de unos rasgos identitarios. El tratamiento de la vida cotidiana y los acontecimientos locales confiere al museo un nuevo papel: el de institución implicada en la creación de identidad.

La mirada de los museos etnológicos sobre la cultura popular implicará una serie de fases caracterizadas por mantener un posicionamiento diferencial sobre el contenido del mismo concepto de cultura popular. Desde este punto de vista y siguiendo la clasificación que Prat hace de este concepto, podemos establecer, sin ser exhaustivos, cuatro grupos de museos:

1. Los museos al aire libre y los heimatmuseos que, en una primera fase, se caracterizan por la asociación que hacen entre cultura popular y tradición, recogiendo las diversas manifestaciones de los estilos de vida preindustriales y generando toda una serie de oposiciones de tipo temporal (tradicional/moderno) y espacial (rural/urbano) que ya habían caracterizado el trabajo de los folkloristas. Posteriormente y por la propia temática tratada estos mismos museos construirán la base de la relación entre patrimonio etnológico e identidad.

2. Los museos de artes y tradiciones populares y, también los museos de los países descolonizados, entienden la cultura popular como cultura nacional y generan unas oposiciones del tipo (españoles/ catalanes-gallegos/valencianos).

3. Los museos comunitarios, que operan con una idea de cultura popular más estrechamente ligada al modelo italiano basado en una 
oposición de clase social (cultura dominante/cultura subalterna) que mantienen una actitud crítica hacia los desajustes económicos y sociales provocados por el sistema capitalista y el estado moderno. Son museos que surgen a finales de los años 60 para ofrecer a los grupos sociales desfavorecidos la posibilidad de acceder a su propia cultura e incitar a asumir su futuro (Duclos y Vieillard, 1992).

4. Los ecomuseos, museos de civilización y los museos de sociedad incluyen una serie de elementos presentes en las anteriores etapas: trabajos desde la óptica de la tradición, la cultura nacional y la clase social. La diferencia, en este caso, se establece a partir del uso del plural en estas nociones. Es decir, no pretenden recoger una sola tradición, una sola identidad nacional o una visión desde una sola clase social, sino que el museo representa la diversidad cultural ya sea de un territorio, una región, una nación o bien de la misma idea de humanidad.

Con la aparición de la temática popular dentro del museo se produce un nuevo encuentro entre antropología y patrimonio. La antropología y el museo etnológico encuentran una misma justificación de su existencia: la urgencia. El "salvamento etnográfico" ha sido un tema recurrente tanto en antropología como en el mundo del patrimonio para justificar una serie de prácticas y actuaciones sobre la cultura popular en detrimento de otras manifestaciones culturales más contemporáneas y actúales trata de "salvar", sobre todo, los testimonios de un estilo de vida rural, gravemente amenazado por los efectos uniformizadores de la revolución industrial.

Desde el punto de vista patrimonial esta idea fue, por ejemplo, uno de los principales ejes de la creación de los museos al aire libre de los países escandinavos. El general Adolphe Hoefer (impulsor del Nederlands Openlucht Museum-1918) expresó esta visión, el año 1912, de la siguiente manera:

Pero quien rechazaría lo cotidiano por lo que es nuevo y monótono?. Aquí y allá la gente utiliza muebles y herramientas que aún son características de su región, pero poco a poco los productos uniformes de las fábricas se van introduciendo. (De Jong, 1994, p. 35-36).

Uno de los primeros museos al aire libre fue el de Skansen (Suecia), creado por A. Hazelius (1891). El modelo propuesto por Fri- 
luftsmuseet Skansen sentó las bases y los principios generales de los primeros museos al aire libre que, a finales del siglo XIX y principios del siglo XX, aparecieron en la mitad norte de nuestro continente y que se pueden resumir en los siguientes puntos: a) se ocupan de los estilos de vida preindustriales, pero, a diferencia de otros museos con la misma vocación, en este caso el ámbito del museo no es un edificio, sino un espacio donde encontramos varias construcciones del mundo rural, pertenecientes a lugares y épocas diferentes; b) desarrollan una serie de actividades relacionadas con la artesanía, la recuperación de antiguos oficios, la reproducción de diferentes fases de los procesos de trabajo y las fiestas de temática rural.

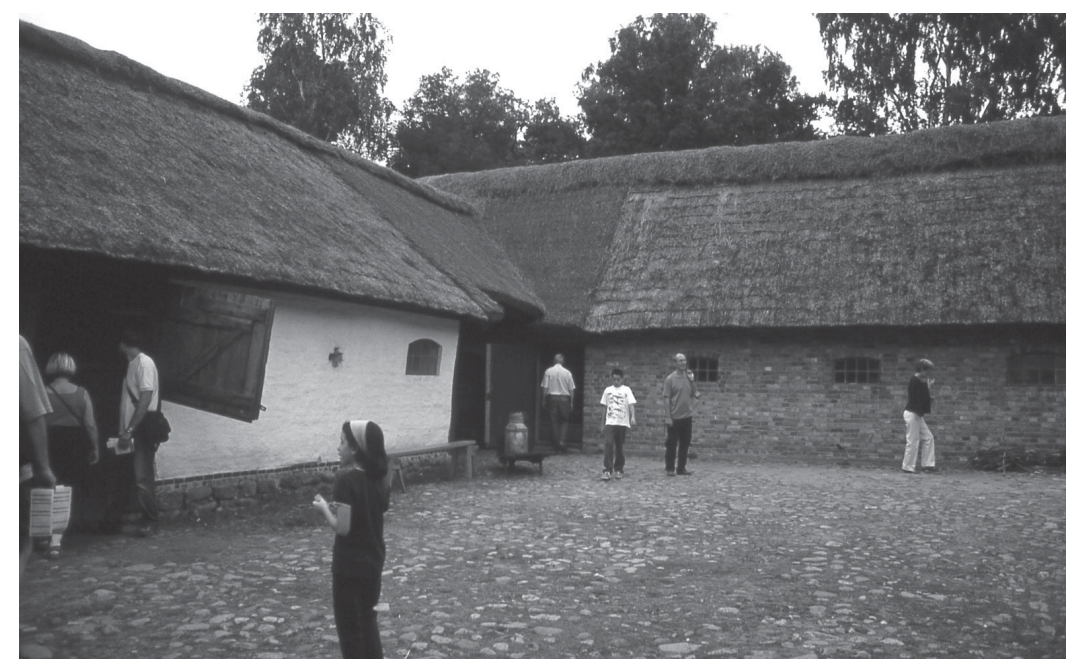

Foto 1: Detalle de una granja en el museo al aire libre de Skansen Fonte: Agustí Andreu Tomàs

Los museos al aire libre se convertirán en los precursores de una mirada que perseguirá poner de relieve la identidad cultural de un determinado territorio. El museo se convertirá en una institución política en un momento en que la nación toma conciencia de sí misma como nación (Nora, 1986). El desarrollo de determinados museos al aire libre se inserta precisamente en este contexto político de creación de conciencia e identidad nacional/regional en que están inmersos la mayoría de los países escandinavos en esta época (Maure, 1993). 
Este aspecto que acabamos de citar, el patrimonio como fuente de creación identitaria, se convirtió también en el eje central de toda una serie de museos que surgieron en Alemania bajo la denominación de Heimatmuseus. Básicamente estos museos alemanes elaboraron un discurso que pone su énfasis en dos aspectos clave, que luego encontraremos en el mensaje elaborado, si bien con otras connotaciones, desde el ecomuseologia: los aspectos identitarios y una museología orientada a la comunidad local .

El museólogo mexicano, Alfredo Cruz-Ramírez (1985) ha sido uno de los primeros autores en reivindicar claramente la posición privilegiada de este tipo de museos dentro del 'árbol genealógico' de los ecomuseos, aspecto éste que según el autor antes citado, ha sido totalmente olvidado y en otros casos silenciado, con motivo de la estrecha relación que existió entre los heimatmuseus y el régimen nacional-socialista.

En su origen, los heimatmuseus, nacen con una clara vocación de utilizar el patrimonio como herramienta para crear en los individuos un sentimiento de vinculación a la patria. El punto de partida es el mismo que en el caso de los museos al aire libre, el patrimonio sobre el que trabajan estos museos gira en torno a la temática popular y tradicional, de la que son depositarios los agricultores y que, con el nacimiento del proletariado industrial y los procesos de desarraigo inherentes al fenómeno de la industrialización, está gravemente amenazada. La focalización de los trabajos y los esfuerzos de los heimatmuseus sobre las tradiciones populares permitirá, en definitiva, preservar la verdadera cultura germánica y luchar contra la influencia uniformizadora de la civilización industrial:

Avec la naissance du prolétariat industriel, le "peuple" et ses "traditions" devinrent un object de recherche en soi. En fait on ne se pencha pas, sauf dans de rares cas, sur le trevailleur industriel, mais bien plutôt sur son image inversée, l'habitant des campagnes...La presentation de l'histoire) régionale en tant que facteur constitutif de l'histoire nationale n'est pas sans avoir joué un rôle important dans les musées allemands de la fin du siècle. Ces fonctions identitaires dévolues au musée contribuèrent à en faire le garant de la "culture" 
germanique face a l'influence de la "civilisation". (Roth, 1989, p. 129-130)

Esta vinculación entre patrimonio etnológico y cultura popular, tendrá un nuevo impulso con la creación en Francia, por parte de Paul Rivet y Georges-Henri Rivière, del museo de Artes y Tradiciones Populares (MATP).

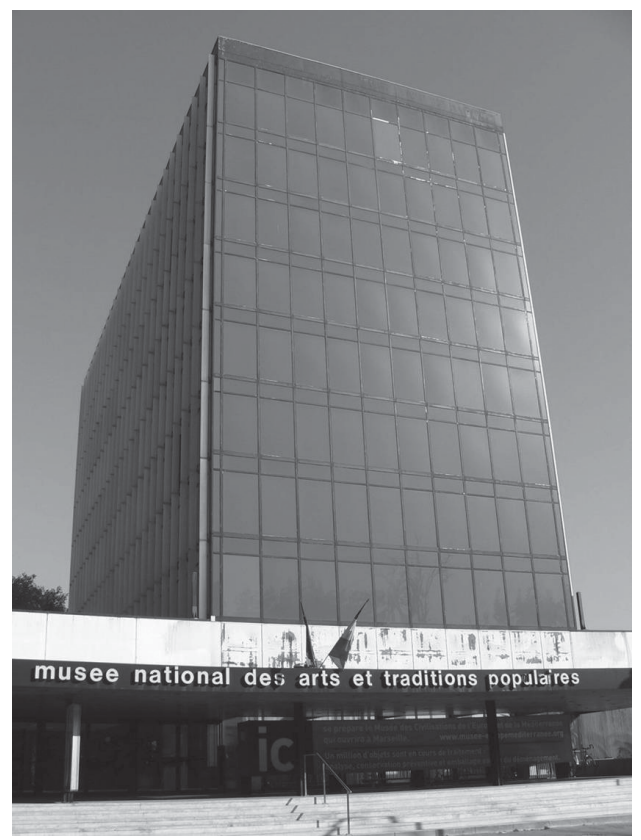

Foto 2: Edificio del Musée National des Arts et Traditions Populaires, cerrado en el año 2005

Autor: Agustí Andreu Tomàs

Paul Rivet y Georges Henri Rivière conciben un museo donde la investigación debe tener un papel fundamental, esta afirmación la sustentan en dos ideas básicas: el museo-laboratorio y el objeto testimonio. Estos dos museólogos franceses se centraran en uno de los aspectos clave de la museología etnológica, la idea de objeto testimonio (desde testimoniar un estadio evolutivo, una totalidad cultural o una identidad local) y que implica que se han restituir las coordenadas sociales, económicas y simbólicas de los objetos. Desde la etnología el objeto patrimonializado sólo tiene sentido en una red o conjunto de relaciones simbólicas de las que es el producto y a la vez el testi- 
monio (Fabre, 1997). El proyecto de Rivet y Rivière concibe el museo de etnografía como un conservatorio y a la vez como un observatorio cultural, como un centro de investigación y documentación y como un centro de conservación y exposición. Los servicios de investigación adquirirán una existencia formal en el año 1966 con la creación del Centro de Ethnologie Française, laboratorio asociado al CNRS.

Desde los años sesenta el interés de Georges Henri Riviére se centró no ya en un determinado tipo de patrimonio, la arquitectura rural, sino en un espacio patrimonial, en un territorio concebido como entidad social, cultural y territorial que implicará la conservación "in situ" de los elementos patrimoniales y que, por Isaac Chiva significa la definitiva ruptura con el mundo del folclore y el paso a un acercamiento etnológico sobre el patrimonio:

Sourtout, en remplaçant l'observation des objets et traits culturels isolés, par la saisie de ces faits sociaux totaux que sont les groupes et territoires régionaux, cet hors pair aurà marqué, dans la connaissance de la société française, les passage du floklore a l'ethnologie (Chiva,1985, p.15).

Este acercamiento etnológico, a finales de los años 60, se concretará en la creación de un nuevo tipo de museo, el ecomuseo, que vinculará estrechamente a esta institución con un territorio y su población y contribuirá a superar la crisis en la que se encuentran inmersos los museos, especialmente aquellos que se ocupan del patrimonio cultural.

\section{Los ecomuseos y la nueva museología}

A mediados del siglo XX se produce una crisis y un agotamiento de las teorías museológicas tradicionales. La superación de esta crisis conllevará la aparición de unas nuevas formas de trabajar y de entender el patrimonio y los museos. En este sentido podemos hablar de dos nuevas vías: a) la vía angloamericana (finales de los años 50): los centros de interpretación; y b) la vía francesa (años 60): los ecomuseos. Ambas vías comparten unas mismas características: un origen vinculado a las políticas institucionales de protección de la naturaleza, a la aparición y desarrollo de los parques nacionales (Estados Unidos/vía interpretativa) y a la creación de los parques naturales (Francia/vía eco- 
museística) y, también coinciden, en que las activaciones patrimoniales que proponen centran su interés en la búsqueda de la participación de la población y se implican en el desarrollo económico y social del territorio: convierten el patrimonio en un instrumento de desarrollo.

La vía interpretativa tuvo una gran importancia en el mundo anglosajón sobre todo en las instituciones de protección de la naturaleza, los servicios pedagógicos de educación ambiental y en la conservación "in situ" de los elementos del patrimonio cultural y natural. La vía ecomuseística implicó una "revolución" desde dentro de la misma institución museística y sus planteamientos tendrán una mayor incidencia en el concepto y valorización del patrimonio así como en la función social que deben llevar a cabo los museos.

Entre otros aspectos, los ecomuseos fueron posibles gracias a que el gobierno francés creo la delegation pour l'Aménagement du Territoire et l'Action Régionale (DATAR). Este organismo fue el encargado de elaborar el concepto de parque natural regional, definiéndolo como un instrumento para luchar contra el empobrecimiento económico, social y demográfico de determinados territorios rurales a través de la utilización del patrimonio natural y cultural. Se trataba de crear una institución capaz de dinamizar económicamente el sector rural a través de la delimitación de una serie de áreas naturales que sirvieran de equilibrio entre los espacios urbanos y rurales. El desarrollo económico propuesto por estos espacios naturales se centraba, sobre todo, en impulsar y canalizar el flujo turístico interior. Básicamente se pretendía reorientar el turismo interno hacia aquellas zonas rurales más deprimidas (un 40\% de los franceses pasaba tres semanas fuera de casa) y aprovechar también el turismo exterior (en el 1965 Francia recibía 7 millones de turistas extranjeros). El primer parque natural francés se creó en el año 1968, el PNR Saint-Amand-Raismes con 12.000 ha. cerca del eje metropolitano Lille-Roubaix-Tourcoing. En 1997 ya había 20 PNRF y, en nuestros días la cifra alcanza los 46 PNRF.

En 1966, el grupo interministerial encargado de definir la política de los parques naturales regionales franceses, organizo las "Journés d'études sur les Parcs naturels régionaux", en las que el museólogo francés Georges Henri Rivière fue invitado a participar en la comisión 
dedicada a asesorar a la política de estas instituciones en los aspectos patrimoniales. En esta época el pensamiento de este museólogo francés estaba muy cercano al concepto de museo al aire libre y, por tanto, la utilización del patrimonio arquitectónico como pieza clave de la acción museográfica. Por este motivo una de las primeras propuestas de actuación que se definieron consistió en la creación, dentro de los parques naturales regionales franceses, unos musas de maisons, es decir: trasladar los edificios rurales más representativos y emblemáticos de las diferentes zonas de el área protegida y explotarlos museográficamente. Esta estrecha colaboración entre profesionales del patrimonio cultural y natural propiciará el nacimiento de las dos realizaciones museográficas que se pueden considerar como el punto de partida de los ecomuseos.

La primera de ellas, en 1968, año en que Jean Pierre Gestión, pondrá en práctica las ideas surgidas en los debates generados en torno a los parques naturales con la inauguración de la Maison des Techniques et Traditions Ouessantines, a l'île d'Ouessant, en la bretaña francesa. Los planteamientos museográficos consistieron en utilizar dos casas de la comunidad de Niou Huella. Una de las casas se utilizó para presentar una síntesis histórica de la isla, integrando en su discurso expositivo las principales disciplinas de las ciencias naturales y humanas. La otra casa, en un buen estado de conservación y con todo el mobiliario interior completo, se conservó en su estado original para mostrar el espacio doméstico característico de Ouessant el siglo XIX, como se comentava en la pàgina web del Parc naturel régional d'Armorique:

En France, il fut la première expérience muséographique prenant en compte de façon globale un patrimoine lié à un espace de vie, s'attachant à présenter un specimen de l'habitat traditionnel, dans son environnement immédiat et dans son insertion au tissu des villages et quartiers.

La segunda realización se concretó, igualmente al amparo de un parque natural, en este caso en la zona de las Landas de Gascogne. En 1969 se inauguró el primer museo que en Francia utilizo la denominación de museo al aire libre, el Musée ave plein air des Landes de Gascogne, convertido más tarde en el Écomusée de la Grande Lande. 
El proceso es, pues, similar al de la Île de Ouessant, la intención de la Datar de crear un parque natural en la reactivará unos viejos proyectos museísticos sobre este territorio que datan de los años 30.

En 1943 ya existe un proyecto de creación de un "musée de maisons" en Hossegor, lugar donde se trasladarían, pieza a pieza, los tipos más significativos de construcciones rurales de la región. Para llevar a cabo la materialización del proyecto el parque natural adquirió la propiedad de una casa de la Gran Lande y se dispuso a adquirir otras construcciones para trasladarlas a este espacio. Esta idea al final no se llevó a cabo. El motivo lo encontramos en el hecho de que la utilización, en el proceso de documentación de la casa de Gran Lande, del catastro de 1836 de la comunidad de Sabres hizo cambiar de idea a Rivière.

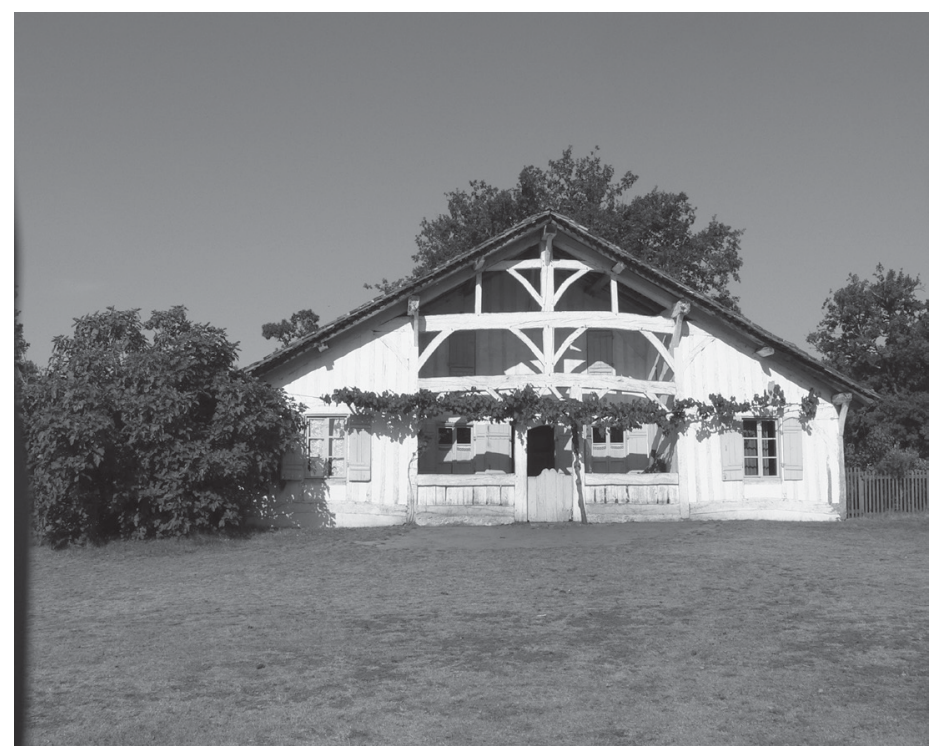

Foto 3. Casa de la explotación agrícola de Marquèze, Écomusée de la Grande Lande Autor: Agustí Andreu Tomàs

Este documento proporcionó una información completa de la estructura de este espacio, permitiendo localizar las diferentes edificaciones que integraron el quartier de Marquèze y Riviére planteo la idea de reconstruir el espacio tal como era en aquella época:

On transfère sans doute des bâtiments, mais ils sont parfaitament identiques à ceux qui avaient disparu et 
sur l'emplacement desquels ils sont reconstruits. L'espace du quartier de Marquèze redevient ce qu'il avait été, avec ses maisons et ses bâtiments d'exploitation, mais aussi ses champs et forêts. Musée au plein'air certes, sans être pourtant un simple musée de maisons, puisque, à travers l'environnement reconstitué, il traite aussi des relations entre l'homme et son milieu.(Hubert, 1989, p. 48).

François Hubert, en el texto anterior, indica le implicaciones de esta idea. Aunque la actuación museográfica continua teniendo el nombre de museo al aire libre, ya no se trata del mismo modelo seguido en los países escandinavos. En este caso lo que se reconstruye es un paisaje, un espacio tal como era en un momento determinado, en el primer tercio del siglo XIX. Mientras que los museos al aire libre inventan un espacio, a base de concentrar en el una serie de edificios traidos de diferentes lugares, con el fin de hacer "visible" un determinado estilo de vida, Marqueze se museografía un espacio inmovilizándolo en el tiempo.

El proceso de recuperación de este espacio no afectó sólo al patrimonio arquitectónico, también se reconstruyeron los demás elementos integrantes de la explotación agrícola: los campos, el huerto, los bosques ... es decir, el proyecto tomó en consideración tanto el patrimonio cultural como el natural. Ya no se trata de un "musée de maisons", sino de un museo (como las casas de Niou Huella) que asocia los edificios a un paisaje con el fin de presentarnos las relaciones del hombre con el medio. Las experiencias de la Île de Ouessant y de Marqueze abrieron un nuevo horizonte al mundo de los museos, que fue inicialmente continuado por dos nuevas realizaciones dirigidas por dos discípulos de Rivière. En el Parque Nacional de Cévennes, en 1971, Gerard Collin puso en marcha el Ecomuseo de Mont-Lozère y, en 1973, Jean Claude Duclos inauguró la Masía Pont-de-Rousty el Parc Naturel Regional de la Camargue.

El contenido del concepto de ecomuseo fue ampliándose y redefiniendo a través de nuevas realizaciones que profundizaron en determinados aspectos de su filosofía. Entre los años 1971 y 1974, Marcel Evrant (con la participación de Rivière y de Hugues de Varine) creó una nueva experiencia en la comunidad urbana de Le Creusot-Mon- 
tceau-les-Mines. Fue la primera vez que un proyecto ecomuseístico se desarrolló sobre un espacio urbano que englobaba un total de 16 pueblos, con una extensión de $500 \mathrm{Km} 2$ y cerca de 90.000 habitantes. Creusot, en definitiva, significo la ampliación del campo de actuación de los ecomuseos más allá del medio social y natural. El mundo urbano e industrial se suman a las propuestas de esta nueva institución museística. Otro de los objetivos básicos de este nuevo ecomuseo lo constituyó su énfasis en el desarrollo comunitario, en implicar a la población en las diferentes fases del proceso: en su concepción, funcionamiento y evaluación.

El ecomuseo es fruto también de una serie de museos que le precedieron, los museos al aire libre, los heimatmuseus alemanes y los museos comunitarios americanos así como de las diversas aportaciones y reflexiones de diferentes museólogos de todo el mundo y de las diferentes experiencias desarrolladas fuera de las fronteras de Francia y que, aparte de contribuir a enriquecer las ideas ecomuseològiques, contribuirán decisivamente a crear el movimiento de la nueva museología. La práctica ecomuseística, muy brevemente, la podemos caracterizar de la siguiente manera:1) toma en consideración el hombre y la naturaleza, 2) es un museo del tiempo y del espacio, 3) es un museo con un programa interdisciplinario, 4) es un museo implicado en la protección de la naturaleza , 5) es un museo de carácter territorial, 6) está al servicio de la sociedad y de su desarrollo y 7) implica a la población local en su gestión.

El ecomuseo y la nueva museología implican sobre todo un cambio en la manera de concebir el patrimonio etnológico, a partir de ahora, como comenta Jean Claude Duclos en referencia a los museos de sociedad y aplicable también a la dinámica patrimonial instaurada por ecomuseos, “[...] a la cuestión de saber lo que el museo de etnografía debe adquirir se agrega otra, la de saber lo que va a contribuir al desarrollo de aquellos en cuyo nombre hace la adquisición" (Duclos, 2005).

Los parques naturales franceses y los ecomuseos iniciaron lo que podemos llamar la utilización del patrimonio con fines de revitalización económica: 
La 'utilización' empresarial del patrimonio con fines de revitalización económica y social aparece como la punta de lanza de otro tipo de desarrollo, respetuosa con la historia y con el medio ambiente, y de una nueva concepción de la cultura como vector de identidad territorial y soporte movilizador de las diferentes comunidades locales (Innovación y empleo, 1991, p. 1).

Aunque los ecomuseos delimitan un nuevo marco en relación al uso social del patrimonio, la multiplicación de este fenómeno implicará su propia crisis. La mayoría de ecomuseos no fueron capaces, en su intento de representar una cultura ligada a un territorio, de escapar al énfasis y la focalización de sus representaciones sobre la cultura popular y tradicional y el pasado.

\section{Las propuestas de los años 80 y 90 del siglo XX: los museos de civilización y de sociedad}

Para escapar de esta, como le llama Segalen (2005), obsesión por el pasado Roland Arpin y su equipo crearon un discurso museológico nuevo alrededor del Musée de la Civilisation de Québec. Inaugurado en 1988, bajo la denominación de civilización, trata de integrar las diferencias étnicas (diversidad cultural) y las particularidades sociales, territoriales, culturales y religiosas (especificidad / identidad cultural). Es un museo que no se circunscribe, como sus predecesores, a fenómenos limitados a un período histórico determinado ni a un grupo cultural particular. Se trata de un museo que sitúa a la persona humana y no al objeto como centro de sus preocupaciones, que da prioridad a la acción cultural y a su relación con los visitantes, que desplaza la identidad por la diversidad y el objeto por hombre (Veillard,1993). Un museo que no fundamenta su existencia en torno a lo que tenemos (las colecciones) sino en relación a lo que decimos y hacemos (Pais de Brito, 2008).

Al otro lado del Atlántico los museos de etnología se reubicaron bajo una nueva denominación, el de sociedad. Vaillant explica el porqué de la utilización de este concepto para el nuevo renacer de los museos franceses: 
Ce terme est choisi pour rassembler les musées qui partagen le même objectif: étudier l'evolution de l'humanité dans ses composantes sociales et històriques, et transmetre le relais, les reperes pour comprendre la diversité des cultures et des sociétés (Vaillant, 1993, p. 37).

Prado (2005), sintetiza la diferencia entre un museo clásico de etnología y un museo de sociedad a través de las siguientes palabras clave, el primero, es un museo del "autre/aillleurs/hier" mientras que, el segundo, se caracteriza por los términos "proche/ici/modernitécontemporain". Los museos de civilización y los sociedad implicaron una progresiva disminución de la importancia del objeto y de la obsesión por el pasado sustituyéndolos por la idea de hombre y por el interés hacia el presente y su proyección al futuro, así como por la exploración de la diversidad cultural. El paso de la construcción del otro a la construcción del nosotros, junto con los cambios sociales y políticos de la época que se dieron, constituyen el punto de partida de las nuevas reformulaciones de los museos de etnología que son depositarios del patrimonio de los demás, es decir, de aquellos museos que han constituido su fondo a partir del típico modelo colonialista de la sociedad occidental. En algunos casos, como en Europa, esta crítica se realizó desde dentro mismo de la propia institución, mientras que en el resto del mundo, este cuestionamiento fue liderado por las poblaciones autóctonas locales. La voluntad de estas poblaciones para hacer oír su voz dentro del museo provocó un replanteamiento sobre el sentido de las colecciones acumuladas y sobre la imagen de las representaciones que de éstas ofrecía el museo. Otra consecuencia de esta crítica social al museo será la aparición de los museos administrados por los autóctonos.

Los museos europeos que contienen colecciones extra-europeas también redefinirán su mirada sobre el patrimonio etnológico. La mayoría se convertirán en un espacio de diálogo, de reconocimiento entre culturas y se orientarán hacia una reflexión sobre el multiculturalismo y las relaciones interculturales

En las últimas décadas la mayoría de los museos de etnología se han renovado mediante diferentes actuaciones y planteamientos: desde una renovación que afecta a sus políticas de exposición: mayor peso 
de las exposiciones temporales (renovación constante de sus contenidos), creación de exposiciones espectáculo (con un claro dominio del diseño respecto del mensaje), introducción de las nuevas tecnologías (nuevos medios tecnológicos y realidades virtuales), renovación de las mismas colecciones (entrada en el museo del objeto actual, como en el museo al aire libre de los Países Bajos).

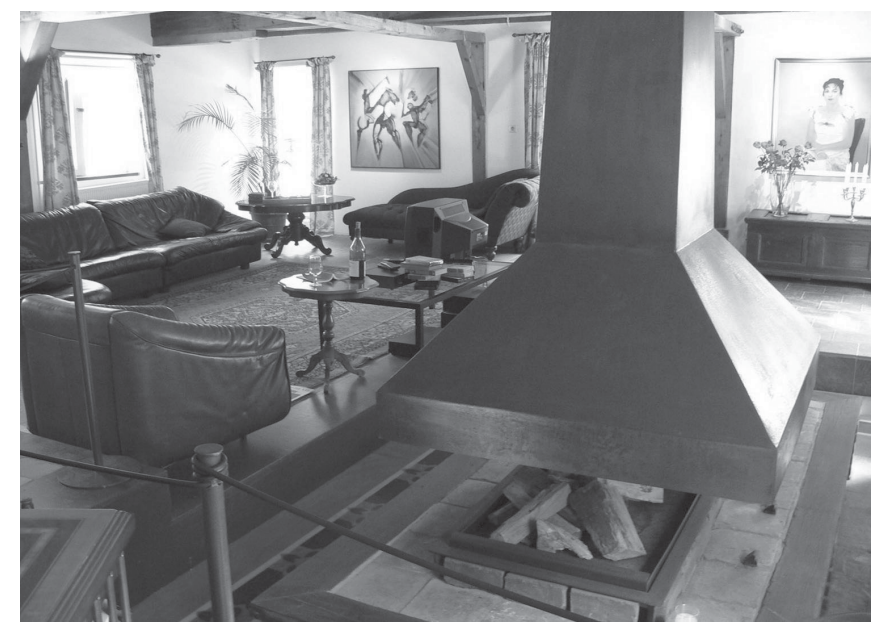

Foto 4: Granja reconvertida en segunda residencia en el Nederlands Openlucht Museum Autor: Agustí Andreu Tomàs

En otros casos esta adaptación ha significado el acercamiento de esta institución a la manera de hacer de los parques temáticos (caso del Écomusée d'Alsace) o bien a partir de la creación de nuevos tipos de museo (como es el caso de los economuseos canadienses, una combinación original de empresa artesanal y museo). 


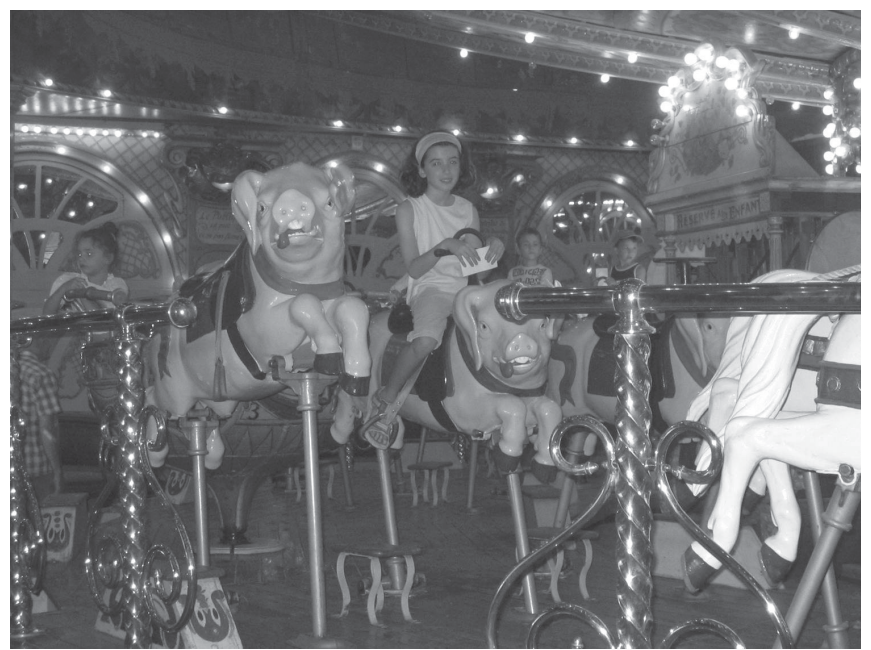

Foto 5: Le carrousel-salon “ Eden Palladium “ en el Écomusée d'Alsace Autor: Agustí Andreu Tomàs

\section{Entre el siglo XX y el siglo XXI: la renovación de los museos a partir de la transformación del patrimonio etnológico.}

Desde el siglo XIX hasta el siglo XX la renovación de los museos de etnología se ha llevado a cabo, también, a partir de diferentes maneras de interpretar el patrimonio etnológico: ya sea para mostrar estadios culturales, áreas culturales, para presentar el progreso técnico, para mostrar las identidades culturales o, entre otros, para explicar la diversidad cultural o hacer un ejercicio de crítica cultural. El museo también se ha redefinido a partir de los cambios en sus funciones sociales: desde la educación y la investigación a la implicación de esta institución en el desarrollo social y económico de un determinado territorio. A pesar de estos cambios que acabamos de citar, aún existe una continuidad en la idea y concepto del patrimonio etnológico. Patrimonio que podemos diferenciar del patrimonio artístico, del científico y técnico, del natural y de cualquier otro tipo. Así, mientras que el patrimonio artístico se centra en el valor estético y el científico y técnico en los conocimientos, los "savoir-faire" y en las técnicas, el etnológico se ha centrado en el valor de uso y en los aspectos simbólicos (creencias, rituales ...). 
La redefinición de los museos de etnología que actualmente se da en Francia implica, en cierto modo, la transformación del patrimonio etnológico en patrimonio artístico y en patrimonio científico y técnico. Lo intentaremos explicar a partir de un museo que ya existe, el Musée de quai Branly, y de dos museos que aún no están inaugurados: el Musée de les Civilisations de l'Europe et la Méditerranée (MUCEM, cuya inauguración está prevista para la primavera de 2013) y el Musée de las Confluences (inauguración prevista para el primer trimestre de 2014).

Lo que, en el año 1996 en París, se anunció como un nuevo museo, Musée de l'Homme, des Arts et des Civilisations, finalmente se ha convertido en un museo que continúa con el mismo nombre que antes (el Musée de l'Homme) y dos nuevos museos (el Musée de quai Branly y el MuCEM). La creación de quai Branly no ha estado exenta de polémica ya que las colecciones etnológicas de dominio extra-europeo se han ubicado en un espacio museográfico dedicado a presentar la vertiente estética de los objetos.

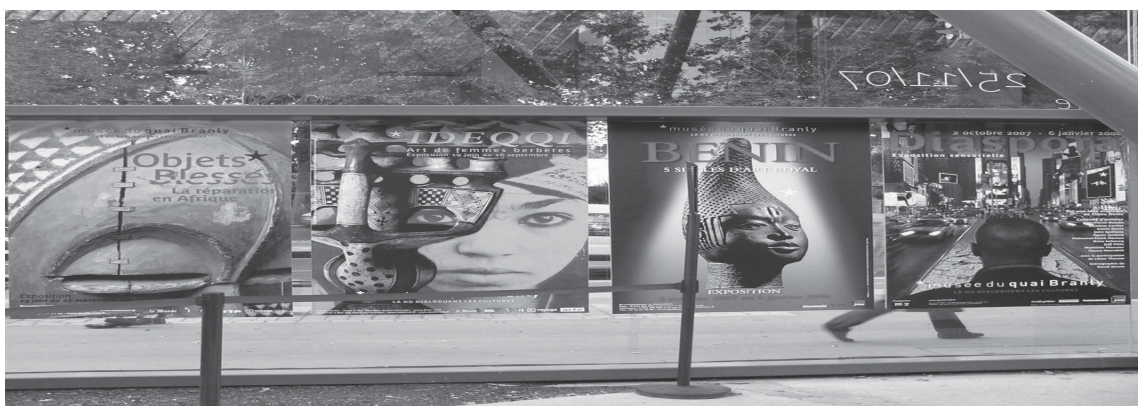

Foto 6: Objets Blessés. La réparation en Afrique, IDEQQI. Art des femmes berberès. BENIN. 5 siècles d'art Royal y Diaspora. Exposition sensorielle.Exposiciones temporales del Musée Quay Branly en 2007 Autor: Agustí Andreu Tomàs

Para los ideólogos de este museo la interpretación etnológica del patrimonio de las sociedades y culturas no europeas está totalmente pasado de moda: no es políticamente correcto hablar de estadios evolutivos, ya sean de tipo cultural (como hacían los evolucionistas) o de tipo técnico (como planteaban las escuelas del materialismo cultural, el marxismo y la ecología cultural) ni tampoco se consideran adecuadas las propuestas de reconstrucción etnográfica (de Boas y Malinowski). 
Tampoco parece que sean de su agrado las soluciones propuestas por otros museos como, por ejemplo, el Musée d'Ethnographie de Neuchâtel, con su museología crítica y de deconstrucción cultural a partir del abandono del fetichismo del objeto y la apuesta por la transculturalidad y la interdisciplinariedad, ni los planteamientos de aquellos museos que se han convertido en un espacio de diálogo y reconocimiento entre culturas diferentes que conviven en un mismo territorio (como, entre otros, el Museo de Etnológico de Barcelona). Finalmente, tampoco han creído conveniente apostar por un discurso que tome en consideración las reivindicaciones de los autóctonos sobre este patrimonio. La apuesta de este museo se ha caracterizado por "mettre en avant la diversié des cultures en l'accent sur l'art". Quai Branly es un museo sobre los "arts premiers" que, como comenta Lévi-Straus (Chiva, 1992) toma por nombre su dirección. Un museo que presenta el patrimonio como obra y no como documento. Las estrategias utilizadas en la exposición permanente para mantener una relación entre el valor estético y el valor de uso (aspectos sociales y simbólicos) de los objetos consisten en una serie de recursos multimedia y virtuales sobre las cuales Clifford opinó lo siguiente:

The overall interpretive strategy minimizes written labels and explanations while aking extensive use of touch-screen video programs. The short programs provide cultural background and show present-day rituals and practices. But it is difficult to connect these performances with the adjacent objects, which seem to occupy a separate time of aesthetic/mystical power and traditional authenticity. One wonders, for example, what connections will made between New Guineacarvings and a film clip showing scores of seminaked men brandishing spears inthe classic, but now dated, ethnographic film Dead Birds (1965). (Clifford, 2007, p. 10)

Si, a partir de quai Branly, asistimos a un proceso de transformación de una parte del patrimonio etnológico en patrimonio artístico, la creación del MuCEM y del Musée des Confluences apuntan hacia otro tipo de transformación que convierte al patrimonio etnológico en patrimonio de la ciencia y la técnica ya que lo presentan como un conjunto de conocimientos, de técnicas y de "savoir-faire". En el 
caso del MuCEM este planteamiento se argumentará a través de una exposición temporal que este museo realizó en el año 2009 y en el caso del Musée des Confluences en base a su proyecto museológico y museográfico.

La reconversión del MATP en MuCEM se inserta dentro de un proceso de europeización de los ATP que pasaran de representar identidades nacionales a dotar de referentes simbólicos la idea de Europa. En esta misma dirección Segalen (2005) comenta que actualmente en Europa estamos asistiendo a la creación de tres museos de ámbito europeo: el Museum Europäischer Kulturen (Mek-Berlin), el Musée de l'Europe (Bruselas) y el MuCEM (Marsella). Con la destitución de M. Collardelle, en 2009, como responsable del proyecto del MuCEM, parece que se ha producido un cambio en la orientación de este museo y su interés se ha desplazado hacia la idea de Mediterráneo, definiéndose como: "un grand projet pour la Méditerranée" (www.mucem. org/). Que un museo de etnología muestre la temática de la identidad no presenta ninguna novedad, lo interesante de este caso es que, en lugar de las clásicas identidades, locales, regionales o nacionales, se representan unas identidades supranacionales (ya sea europea o mediterránea). El MuCEM, y los otros museos europeos que hemos citado, son ante todo, unos museos identitarios que enlazan con la filosofía de los museos etnológicos de finales del siglo XIX y del siglo XX.

El 9 de enero de 2009 se inauguró la exposición "SOS. Save our resources" realizada por el MuCEM con la colaboración de la Fédération des Musées d'Agricultue et du Patrimoine Rural en el marco del programa europeo CULTURE 2000. Esta exposición, de tipo itinerante, se inauguró en el Écomusée de Marquèze (Landes de Gascogne-Francia). Creado en 1969 por Georges Henri Rivière, este ecomuseo se renovó en el año 2008 con unos nuevos espacios de exposición y salas polivalentes que han implicado una actualización del discurso museográfico a partir de la incorporación de un análisis contemporáneo (el presente del territorio y los posibles escenarios de futuro) sobre la zona de las Landes de Gascogne. En esta exposición, consagrada, como tantas y tantas exposiciones de etnología, al patrimonio rural lo interesante es que no se pretende idealizar los estilos de vida del pasado sino de 
inspirarse en ellos. Este planteamiento implica recuperar el patrimonio rural desde una óptica diferente, no como identidad (aspectos simbólicos) sino como "savoir-faire" (aspectos técnicos): "En effet, le patrimoine rural constitue une resource ancrée dans des paysages, des savoir-faire, de techniques ou encore des traditions, donc nos modes de vie actuals peuvent s'inspirer et tirer parti" (Laubrie y Foissey, 2009:3). Se trata de presentar el patrimonio rural como un conjunto de técnicas, conocimientos y habilidades que nos ayuden a repensar nuestra manera de ser y de actuar. Una mirada sobre el patrimonio rural realizada desde las preocupaciones del presente y en base a uno de los mitos de la modernidad: la idea de sostenibilidad y, por tanto, a lo que este patrimonio rural puede aportar (a través, por ejemplo, de la recuperación de antiguas artesanías -aspecto este, por cierto, que ya desarrollaron los ecomuseos- o de técnicas de construcción de la arquitectura popular y tradicional) a la práctica del desarrollo sostenible.

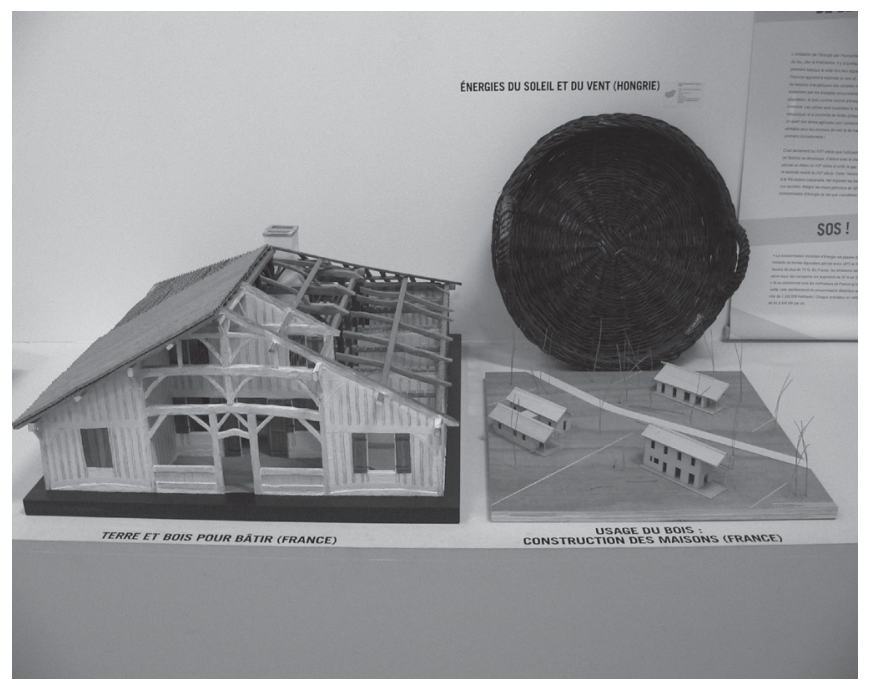

Foto 7: Maqueta de la expocición "SOS. Save our resources". Pabilion Marquèze, Écomusée de la Grande Lande, 2009

Autor: Agustí Andreu Tomàs

Si el MuCEM enfatiza los aspectos tecnológicos de la cultura popular y tradicional, el Musée des Confluences (Lyon) lo hará (2014) con el patrimonio etnológico extra-europeo. Definido como un proyecto cultural para comprender el mundo y como un museo de ciencia 
y sociedad que pretende comprender los mecanismos del desarrollo de las sociedades y su relación con el medio ambiente. El fondo de este museo está compuesto por colecciones de ciencias de la tierra, de la vida, de etnología extra-europea y de la ciencia y la técnica. La presencia de la colección de tipo etnológico (extra-europeo) entre las colecciones de las ciencias de la tierra y de la vida en cierta manera nos remite al origen de la museología etnológica en los Estados Unidos y su desarrollo en los museos de historia natural. Por otro lado la presencia de las colecciones de ciencia y técnica así como los planteamientos expuestos en su página web (www.museedesconfluences. fr/, consultada el 25/08/2011) en los que se destaca que, a través de las colecciones etnológicas, se presentaran exposiciones que permitirán a los visitantes interrogarse sobre las dimensiones técnicas, históricas, estéticas y contemporáneas de la sociedad induce a pensar en una mirada sobre el patrimonio etnológico que sustituye los aspectos culturales y simbólicos por los técnicos (saberes, técnicas,...).Posiblemente estemos, pues, asistiendo a una renovación de los museos de etnología que implica, en algunos casos, la transformación del patrimonio etnológico en patrimonio artístico y en patrimonio de la ciencia y la técnica. Habrá que observar el desarrollo de los planteamientos de los nuevos museos etnológicos que se están creando, así como los cambios que se producen en los museos ya existentes, para poder definir con más precisión las transformaciones -según el diccionario de la Real Academia Española transformar és transmutar algo en otra cosa- de los museos de etnología que se analizan en la última parte de este artículo.

\section{Notas:}

1 Una versión más breve, de 10 páginas, de este artículo fue presentada como comunicación al III Seminario Iberoamericano de Investigación en Museología (SIAM) celebrado la Universidad Autónoma de Madrid los días 27, 28 y 29 de octubre de 2011.

2 N.T.: Professor do Departament d'Antropologia, Filosofia i Treball Social de la Facultat de Lletres-Universitat Rovira i Virgili (Tarragona-España). 


\section{Referencias}

ANDREU, Agustí. De l'objecte etnogràfic al patrimoni etnològic.

Antropologia, patrimoni i museus. Revista Valenciana d'Etnologia, n.3, p. 1341,2008.

BERGERON, Yves. Du musée de l'Homme du Québec au Musée de la Civilisation. Transformations des musées d'ethnographie au Québec.

QuAderns-e de l'Institut Català d'Antropologia, [on-line], n. 9, 2007. Disponible en: http://www.antropologia.cat//antiga/quaderns-e/09/Bergeron.htm > . Acceso en: 10 abr. 2009.

BERGERON, Y. y DUPONT, Luc. Essai sur les tendences dans les musées de société. Le cas du musée de la Civilisation. In: BERGERON, Y. (Dir). Musées et muséologie. Nouvelles frontières. Essais sur les tendences. Québec: Musée de la Civilisation, Société des Musées Québecois, 2005, p. 55-255.

BOUQUET, M. (Ed.) Academic Anthropology and the Museum. Back to the future. Oxford: Berghahn Books, 2001.

CHIVA, Isac. Georges-Henri Rivière un demi-siècle d'ethnologie de la France, Terrain, n. 5, p. 76-83, 1985.

. Un prototype, un constat: les musées d'ethnologie. In: Constituer aujourd'hui la mémoire de demain, Actes du colloque de Rennes. Rennes: Musée de Bretagne et M.N.E.S., 1988.

. Qu'est-ce qu'un musée des arts et traditions populaires. Entretien avec Claude Lévi-Strauss.? Le Débat, n. 70, p. 164-173, 1992.

CLAIR, Jean. Les origines de la notion d'écomusée. In: . Vagues. Une anthologie de la nouvelle museologie. Editions W.M.N.E.S., Mâçon, 1992.

CLIFFORD, James. Quai Branly in process. , n. 120, October, p. 3-23, 2007.

COTÉ, M. Les expositions de référence au musée des Confluences. Les cahiers du Musée des Confluences, n. 1, p. 65-75, 2008.

COURCELLES, P. Un musée autrement. Regards.fr. [2011]. Disponible en: <http://regards.netaktiv.com/article/?id=1986\&q=date:2000-06-01>. Acceso en: 12 mayo 2011.

CRUZ-RAMIREZ, Alfredo. Heimatmuseum: une histoire oubliée. Museum, n. 4/ 148, v. XXXVII, p. 241-244, 1985.

CUISINIER, Jean. Pour des musées de l'homme et de la société: oui, masis lesquels?. Le Débat, n. 70, p. 178-187, 1992.

DE L'ESTOILE, Benoît. Le Goût des autres. De l'exposition coloniale aux arts premiers. París: Flammarion, 2007. 
DESVEAUX, Emmanuel. Le musée du quai Branly au miroir de ses prédéceseurs. Ethnologies, v. 24, n. 2, p. 219-226, 2002.

DUCLOS, Jean-Claude. Pour des musées de l'homme et de la société. Le Débat, n. 70, pp. 174-177, 1992. 2005. . Depuis Rivière... Le Monde Alpin et Rhodanie, n. 1-4, p. 139-150,

DUCLOS, Jean-Claude. y VEILLARD, Jean-Yves. Museos de etnografia y política. Museum, n. 175, p. 120-13, 1992.

FABRE, Daniel. Le patrimoine, l'ethnologie. In: NORA, Pierre. (Dir.). Science et conscience du patrimoine. Paris: Fayard, Éditions du patrimoine, 1997, p. 59-72.

GONSETH, Marc-Olivier, HAINARD, Jacques. KAEHR, Roland. (Dir.). Cent ans d'ethnographie sur la colline de Saint-Nicolas 1904-2004. Neuchâtel: Musée d'Ethnographie de Neuchâtel, 2005.

GORGUS, Nina. Le magicien des vitrines. Paris: Fondation Maison des Sciences de l'Homme, 2003.

HAINARD, Jacques. Le musée cette obsession... Terrain, n. 4, p. 104-110, 1985.

. L'expologia a la seva justa mesura. La museografia al Museu d'Etnografia de Neuchâtel. Mnemòsine, n. 4, p. 57-74, 2007.

HAMY, Ernest Théodore. Les origines du musée d'ethnographie. París: Editions Jean-Michel Place, 1988.

HUBERT, François. Historique des écomusées. In: La muséologie selon Georges Henri Rivière. París: Dunot, 1989, p. 146-154.

. Innovación y empleo. Revista OCDE, n. 7, 1991

KREBS, Anne y MARESCA, Bruno. (Coord.). Le renouveau des musées. Problémes politiques et sociaux. Paris: La Documentation Française, 2005.

LAUBRIE, Edouard de y FOISSEY, Colette. (Dir.). Save our Sources. Petit journal de l'exposition. Parc Naturel Régional des Landes de Gascogne, Musée des Civilisations de l'Europe et de la Méditerranée, Fédération Française des Musées d'Agriculture, 2009.

MAIRESSE, François. E musée, temple spectaculaire. Lyon: Presses Universitaires de Lyon, 2002.

MAURE, Marc. Nation, paysan et Nation, paysan et musée. La naissance des musées d'ethnographie dans les pays scandinaves (1870-1904), Terrain,, n. 20, 1993. . La museologie selon Georges Henri Rivière. París: Dunod, 1989. 
NORA, Pierre. Les lieux de la mémoire. La natión. Paris: Gallimard, 1986.

PADIGLIONE, Vincenzo. La ordenación en los pequeños museos etnográficos: nuevos horizontes teóricos entre antropología y arte.

In:_ . Teoría y praxis de la museografía etnográfica. Actas del Ier. Cogreso Internacional de Museografía Etnográfica. Zamora: Museo Etnográfico de Castilla y León, p. 203-217, 2008.

PAIS DE BRITO, Joaquim. Museos y colecciones etnográficas. Objetos y atribución de sentido. In: . Teoría y praxis de la museografía etnográfica. Actas del Ier. Cogreso Internacional de Museografía Etnográfica. Zamora: Museo Etnográfico de Castilla y León, p. 65-72, 2008.

PRADO, Patrick. L'ethnographie française au musée? Ou un nouveau musée de l'ethnologie de la France?. Terrain, n. 25, p.147-157, 1995.

PRAT, Joan. Folklore, cultura popular y patrimonio : Sobre viejas y nuevas pasiones identitarias. Arxius de Sociología, Universitat de Vàlencia, n. 3, p. 87-109, 1999.

RIVARD, René. La nouvelle palette des musées. The Unesco Courier, [online], n. 52, v. 1, p. 40-42. [2010]. Disponible en: <http://unesdoc.unesco. org/images/001 1/001 146/1 14699f.pdf>. Acceso en: 3 sep. 2010.

ROIGÉ, Xavier. La reinvención del museo etnológico. In: ARRIETA, I. Patrimonios culturales: más allá de la historia y del arte. Bilbao: Universidad del País Vasco, 2007, p. 19-42.

ROTH, Martin. Collectionner ou accumuler? A propos des mussées ethnographiques et historiques régionaux en Allematge et en France Terrain, n. 12, p.125-137, 1989.

SEGALEN, Martine. La vie d'un musée 1937-2005. París: Stock, 2005.

VAILLANT, E. Les musées de sociéte en France: chronologie et définition. In: Actes du Colloque National "Musées et Sociétés" Mulhouse-Ungersheim. Paris: Ministère de la Culture, Direction des Musées de France, 1993. p. 16-38.

VAN GENNEP, Arnold .Quelques lacunes de l'ethnographie actuelle. In: HAINARD, J. y KAEHR, R. (Ed.). Temps perdu, temps retrouve. Voir les chases du passé au présent. Neuchâtel: Musée d'Ethnographie de Neuchâtel, 1985. p. 43-50.

VEILLARD, Jean-Yves. Le musée de la Civilisation du Québec. Un monde en continuité et en devenir. Terrain, n. 20, p.135-146, 1993.

VIATTE, Germain. La museologie au musée du Quai Branly. QuAderns-e de l'Institut Català d'Antropologia, n. 9. [2009]. Disponible en: < http://www. antropologia.cat//antiga/quaderns-e/09/Viatte.htm>. Acceso en: 10 abr. 2009.

Recebido em 05/09/2012

Aceito em 10/10/2012 